\title{
Heroin addiction engages negative emotional learning brain circuits in rats
}

\author{
Stephanie A. Carmack, ${ }^{1}$ Robin J. Keeley, ${ }^{2}$ Janaina C. M. Vendruscolo, ${ }^{1}$ Emily G. Lowery-Gionta, ${ }^{1}$ Hanbing Lu, ${ }^{2}$ George F. Koob, ${ }^{1}$ \\ Elliot A. Stein, ${ }^{2}$ and Leandro F. Vendruscolo ${ }^{1}$
}

IIntegrative Neuroscience Research Branch and ${ }^{2}$ Neuroimaging Research Branch, National Institute on Drug Abuse (NIDA), Intramural Research Program, NIH, Baltimore, Maryland, USA.

\begin{abstract}
Opioid use disorder is associated with the emergence of persistent negative emotional states during drug abstinence that drive compulsive drug taking and seeking. Functional magnetic resonance imaging (fMRI) in rats identified neurocircuits that were activated by stimuli that were previously paired with heroin withdrawal. The activation of amygdala and hypothalamic circuits was related to the degree of heroin dependence, supporting the significance of conditioned negative affect in sustaining compulsive-like heroin seeking and taking and providing neurobiological insights into the drivers of the current opioid crisis.
\end{abstract}

\section{Introduction}

The United States is experiencing an opioid dependence and overdose crisis (1). New conceptual frameworks and therapeutic targets are needed to more effectively treat opioid use disorder (OUD) and curb opioid overdose deaths. Individuals with OUD are hypothesized as compulsively using opioid drugs to avoid the severe negative emotional states (e.g., dysphoria, pain, anxiety, and depression) that are experienced during abstinence (2). In both clinical and preclinical models, environmental stimuli conditioned to these negative emotional states can induce opioid use on their own (3-5). Individuals with OUD report withdrawal symptoms and drug craving when encountering drug-related stimuli (6).

Stimuli that are conditioned to opioid withdrawal may perpetuate and reinstate drug seeking by generating negative emotional states that are relieved by drug taking via negative reinforcement $(2,4,7)$. Identifying the brain circuits that support motivational aspects of conditioned withdrawal may provide insights into the long-term neuroplasticity-based consequences that frustrate therapeutic interventions in OUD (2, 8). Thus, we hypothesized that conditioned withdrawal would engage brain circuitry that is involved in negative emotional learning. In the present study, we found that cue-induced conditioned withdrawal engaged brain emotional systems in a rat model of heroin dependence.

\section{Results and Discussion}

To test our hypothesis, we utilized a behavioral model in which cues were paired with heroin withdrawal-induced negative emotionallike states (4). Rats were first trained to self-administer heroin (60 $\mu \mathrm{g} / \mathrm{kg} / \mathrm{i} . \mathrm{v}$. infusion) in short-access (ShA; $1 \mathrm{~h} / \mathrm{d}$ ) or long-access (LgA; $12 \mathrm{~h} / \mathrm{d}$ ) sessions that were designed to model nondependent, controlled use versus dependent, compulsive heroin use (9). LgA rats

Conflict of interest: The authors have declared that no conflict of interest exists. Copyright: () 2019, American Society for Clinical Investigation.

Submitted: October 11, 2018; Accepted: March 19, 2019; Published: May 13, 2019.

Reference information: / Clin Invest. 2019;129(6):2480-2484.

https://doi.org/10.1172/JCl125534. rapidly escalated their heroin intake, whereas ShA rats exhibited stable drug intake (Figure 1A).

In the conditioning phase, rats were treated with saline or naloxone $(120 \mu \mathrm{g} / \mathrm{kg}$, s.c.) 30 minutes into each heroin selfadministration session. Naloxone competes with heroin at $\mu$-opioid receptors and, in this dose range, precipitates motivational signs of withdrawal (e.g., place aversion, increased intracranial self-stimulation thresholds), but not somatic signs of withdrawal (e.g. "wet dog" shakes) in opioid-dependent rats $(4,9-11)$. The treatments were paired with distinct olfactory cues (lemon- or vanilla-scented bedding) in the self-administration chamber. The cue pairings lasted 30 minutes to coincide with the short-acting pharmacological effect of naloxone. After the cue pairing, ShA rats were returned to their home cages and LgA rats completed their 12-hour session without olfactory cues (i.e., with unscented bedding).

Naloxone treatment increased heroin intake relative to saline treatment in both self-administration groups (Figure 1B). Heroin intake remained stable across cue pairings (Supplemental Figure 1; supplemental material available online with this article; https:// doi.org/10.1172/JCI125534DS1). As naloxone has a greater affinity for the $\mu$-opioid receptor than heroin, naloxone likely immediately produced opioid withdrawal in LgA rats. This withdrawal effect might have been relieved by the elimination of naloxone concomitantly with increases in heroin self-administration. An alternative explanation for the increase in heroin intake during naloxone treatment may be that the maintenance of a hedonic tone is disrupted by naloxone and that rats that self-administer more heroin have increased tolerance to heroin.

Earlier work demonstrated that presentation of a compound auditory and visual cue previously paired with naloxone treatment increased intracranial self-stimulation thresholds and motivated heroin intake during heroin self-administration in LgA rats, suggesting that the cue became conditioned to naloxone-precipitated withdrawal (4). Here we confirmed that an olfactory cue previously paired with naloxone similarly increased heroin intake in LgA rats, but not ShA rats, when presented in the absence of naloxone during heroin self-administration (Supplemental Figure 2). Additionally, presentation of an olfactory cue previously paired with naloxone 
A

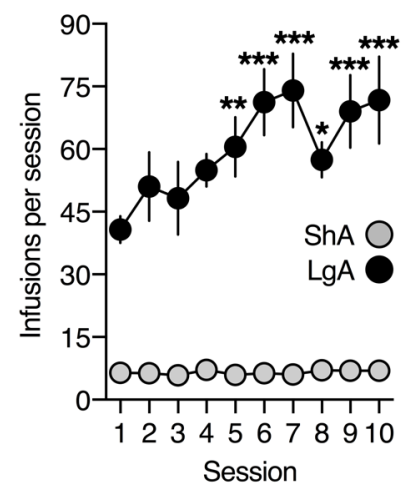

B

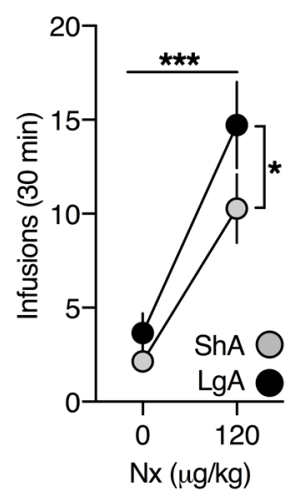

C

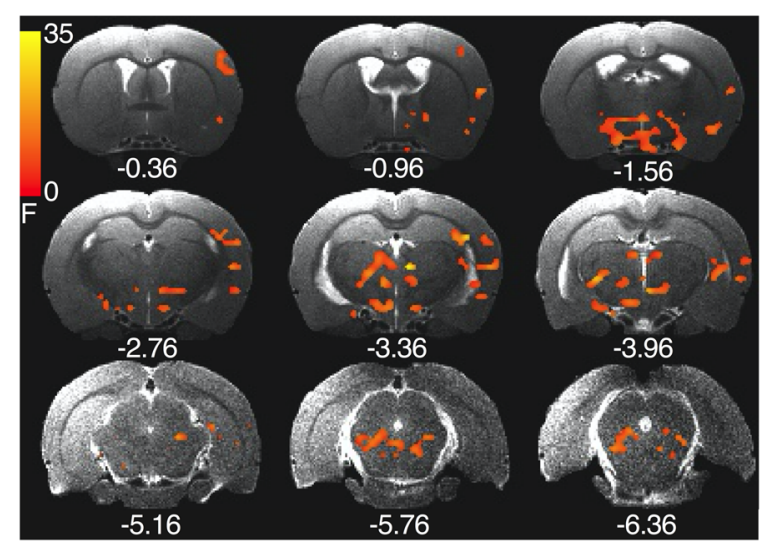

Figure 1. Conditioned heroin withdrawal engages negative emotional learning neurocircuits. (A) Heroin intake during ShA and LgA self-administration sessions. A significant heroin-access $\times$ session interaction was observed $\left(F_{9,171}=4.25, P=0.002 ; 2\right.$-way repeated-measures ANOVA). (B) Heroin intake following saline $(0 \mu \mathrm{g} / \mathrm{kg}$, s.c.) or naloxone ( $\mathrm{Nx})(120 \mu \mathrm{g} / \mathrm{kg}$, s.c.) treatments during cue pairings, presented as the average of the 4 cue pairings per treatment. Significant main effects of treatment $\left(F_{1,19}=35.5, P<0.0001\right)$ and heroin access $\left(F_{1,19}=4.215, P=0.05\right)$ were found ( 2 -way repeated-measures ANOVA). (C) Statistical map ( $F$ values) of the cue $\times$ heroin-access BOLD signal interaction following whole-brain 3-way ANCOVA, with respiration as the covariate $(P<0.01$; 233 voxels, corrected for multiple comparisons). The upsampled (to anatomical images) statistical map is superimposed on anatomical coronal images from a representative subject. Below each section is the anterior-posterior distance from bregma (in mm). Data represent mean \pm SEM. ${ }^{*} P<0.05$; ${ }^{* *} P<0.01$; ${ }^{* *} P<0.001$ (different from session 1 and corrected for multiple comparisons in $\mathbf{A}) . n=11$ ShA rats; $n=10$ LgA rats.

in LgA rats produced greater reinstatement of heroin seeking after extinction compared with presentation of an odor previously paired with saline (Supplemental Figure 3).

To explore the brain circuits that underlie conditioned withdrawal, we presented the olfactory cues alone to lightly anesthetized rats that were subjected to spontaneous heroin withdrawal during functional magnetic resonance imaging (fMRI) blood oxygen level-dependent (BOLD) signal acquisition 24 hours after their last cue conditioning self-administration session (Supplemental Figure 4). The light anesthetic regimen used is known to maintain neurovascular coupling and preserved odor-specific sensory processing $(12,13)$ (Supplemental Figures 4 and 5). The cues were presented in a counterbalanced, blocked design in sequential scans, and respiration rates fluctuated in response to cue presentation (Supplemental Figure 4).

Using the percentage change in the BOLD signal from baseline in response to cue-only presentation as the dependent measure and respiration as the covariate, a whole brain 2 (cues, saline versus naloxone paired) $\times 2$ (heroin access, ShA versus $\operatorname{LgA}) \times 2$ (cue presentation block, blocks 1 and 2 versus blocks 3 and 4) ANCOVA yielded a significant cue $\times$ heroin-access interaction (Figure 1C and Supplemental Figure 4). The large cluster was segregated into 19 anatomically defined regions of interest (ROIs) using a spatially aligned rat atlas (14) (Table 1 ).

We then evaluated the relationship between activation in each ROI and the number of heroin infusions during naloxone plus cue conditioning sessions (shown in Figure 1B); the latter was used as an index of withdrawal severity. After correcting for multiple comparisons, BOLD activation was associated with withdrawal severity in 2 ROIs: (a) a hypothalamic cluster that made up the paraventricular nucleus of the hypothalamus (PVN) and ventromedial hypothalamus (VM) (Figure 2A) and (b) amygdala nuclei that made up the medial amygdala, central nucleus of the amygdala, and extended amygdala (Figure 2D). In both regions, the naloxone-paired cue increased the BOLD signal in $\operatorname{LgA}$ rats and decreased the BOLD signal in ShA rats (Figure 2, B and E). Heroin intake during naloxone plus cue conditioning (Figure 1B) was correlated with both the hypothalamic cluster (Figure 2C) and the amygdala (Figure 2F) BOLD response to the naloxone-paired cue in both self-administration groups. Greater withdrawal severity during conditioning was associated with greater activation in these regions during conditioned withdrawal.

Opioid withdrawal activates extrahypothalamic (e.g., extended amygdala) emotional systems in opioid-dependent rats $(11,15)$, and opioid dependence alters amygdala connectivity in humans (16). Opioid withdrawal also is known to potently activate the hypothalamic-pituitary-adrenal (HPA) arousal/stress axis in opioid-dependent humans and rats $(17,18)$. Activation of the PVN during opioid withdrawal and subsequent driving of the HPA axis may be an early dysregulation that is associated with excessive opioid intake. We hypothesize that dysregulation of the HPA axis and sensitization of the extended amygdala maintain negative emotional states via glucocorticoid signaling $(19,20)$. These results suggest that previously neutral stimuli gain motivational value when paired with opioid withdrawal, first by a hormonal stress-like response, which in turn activates extrahypothalamic brain stress systems in the extended amygdala, forming a pathway for negative emotional states that drive craving and relapse in humans (19-21).

Several other regions exhibited activation patterns that were similar to those seen in the hypothalamic cluster and amygdala (Table 1). Many of these regions have been implicated in emotional learning and are hypothesized to be dysregulated in addiction $(2,22,23)$. These include the lateral hypothalamus, dorsomedial nucleus of the thalamus, ventrolateral thalamus, and dorsal striatum. Along with the amygdala, these regions are activated by heroin cues in individuals with OUD $(22,23)$. The extended amygdala promotes both positive and negative emotional states via its downstream connections to such areas as the lateral hypothalamus (24). 


\section{Table 1. Mean percentage change in BOLD signal from baseline in response to saline and naloxone-paired cue-only presentation for ShA and LgA rats in ROls}

\begin{tabular}{lcccc} 
& \multicolumn{2}{c}{ ShA } & \multicolumn{2}{c}{ LgA } \\
ROI & Saline cue & Naloxone cue & Saline cue & Naloxone cue \\
Amygdala nuclei & 1.3 & -1.1 & 0.1 & 0.9 \\
Anterior hypothalamus & -0.4 & -1.5 & 0.7 & 2.0 \\
Auditory cortex & 0.1 & -1.0 & -0.5 & 0.3 \\
Dorsomedial thalamus & 0.5 & -0.6 & -0.1 & 0.4 \\
\hline Dorsal striatum & -0.1 & -1.1 & -1.1 & 0.6 \\
Ectorhinal cortex & 0.5 & -1.1 & -1.0 & 0.2 \\
Habenula & -0.6 & -0.6 & 1.0 & 0.1 \\
Hippocampus & -0.3 & -0.7 & -0.4 & 0.0 \\
Insula & 0.1 & -1.0 & -0.5 & 0.1 \\
Lateral hypothalamus & 1.3 & -1.1 & -0.3 & 0.4 \\
Lateral geniculate & 0.3 & -0.7 & -1.4 & 0.2 \\
PAG and PVG & -0.2 & -1.4 & -0.5 & 0.5 \\
Peduncle & -0.4 & -3.5 & -1.9 & 0.5 \\
Precommisural nucleus & 0.6 & -0.4 & -0.1 & 0.5 \\
Pretectal nucleus & -0.1 & -1.1 & -0.8 & 0.6 \\
PVN/VM hypothalamus & 1.7 & -1.1 & 0.1 & 1.1 \\
\hline Reticular formation & 0.2 & -1.4 & -1.0 & 0.2 \\
S1/S2 & 0.1 & -0.6 & -0.1 & 0.6 \\
Ventrolateral thalamus & 0.5 & -1.2 & -0.8 & 0.3 \\
\hline
\end{tabular}

We anatomically segregated the cluster defined by the statistically significant heroin-access (ShA versus LgA) $\times$ cue (saline paired versus naloxone paired) interaction (shown in Figure 1C) into 19 ROIs using a standard rat atlas (14). S1/S2, primary and secondary somatosensory cortex.

As part of a habit-learning circuit, the thalamus and dorsal striatum are implicated in reward and incentive salience $(2,25)$. Additionally, the naloxone-paired cue activated the precommissural nucleus (PRC), periaqueductal gray (PAG)/periventricular gray (PVG), and the pretectal nucleus in LgA rats, whereas these same regions were deactivated in ShA rats. The saline-paired cue deactivated these regions in LgA rats. The PRC and PAG/PVG are anatomically connected to the extended amygdala, VM hypothalamus, and pretectal nucleus and play a key role in negative emotional learning $(5,26)$.

Finally, both odor cues activated the anterior hypothalamus and a region consistent with the habenula in $\operatorname{LgA}$ rats, whereas opposite effects were observed in ShA rats. The habenula can inhibit the mesolimbic dopamine system and modulate emotional and motivational states (27). Together with the hypothalamus and amygdala, the habenula serves to maintain hedonic homeostasis $(2,19,26)$. Although the anatomical resolution of fMRI and the small size of the rat habenula limited our ability to confirm its activation, potential engagement of the habenula during negative conditioned responding warrants further investigation.

We found that conditioned heroin withdrawal motivated heroin intake and engaged brain regions that are associated with negative emotional learning, particularly extrahypothalamic and hypothalamic stress/arousal circuitries. These circuits are consistent with and extend fMRI findings in individuals with OUD on drug cue reactivity tasks $(22,23)$. Thus, we argue that conditioned cues can maintain compulsive drug use by removing aversive states (conditioned negative reinforcement) as well as by producing positive incentive states (conditioned positive reinforcement) $(2,22$, 28) and that both forms of learning contribute to allostatic changes in emotional processes that perpetuate opioid addiction.

Exposure to conditioned withdrawal stimuli may drive craving and provoke relapse in individuals with OUD by inducing a powerful aversive stress state that is relieved by opioid use $(7,29)$. The stimuli that trigger conditioned withdrawal are likely the same as those that convey learned tolerance (30). Thus, individuals with OUD are especially susceptible to overdose death when they use opioids in unfamiliar contexts or with unfamiliar cues (e.g., different administration procedure or opioid) and their bodies fail to engage learned compensatory mechanisms (30) or when they encounter conditioned withdrawal stimuli and their drug tolerance is reduced, such as following detoxification in a treatment facility or release from incarceration (31). Critically, the 3 United States Food and Drug Administration-approved medications for OUD, which target opioid receptors (8), may not fully alleviate cue-conditioned withdrawal.

Therefore, we propose that understanding and targeting the brain circuits that underlie conditioned withdrawal and downstream emotional circuitries (e.g., brain stress circuits) provides an innovative conceptual framework for novel treatment and the prevention of opioid overdose deaths. Examining fMRI, and in parallel, psychological responses to drug-related cues provide a potentially powerful approach to understanding individual differences leading to and maintaining compulsive opioid use. Indeed, dysfunction in brain circuits of negative emotional learning is a potential biomarker for tracking progression and remission of OUD.

\section{Methods}

Further information can be found in Supplemental Methods and Supplemental Figures 1-5.

Subjects. Adult male Long-Evans rats (Charles River Laboratories) were group housed (2 to 3/cage) on a 12-hour light/12-hour dark cycle at the NIDA animal facilities in the Biomedical Research Center. At approximately 6 weeks of age (250-275 g), rats were implanted with chronic indwelling i.v. catheters in the right jugular vein under isoflurane anesthesia, as previously described (9). Rats underwent MRI scanning between 3 and 3.5 months of age $(32,33)$. Standard rat chow and water were available ad libitum in home cages and throughout the self-administration experiments, but not during the other experimental procedures. The plurality of experimental procedures was performed in the dark cycle, with few extending into the light cycle.

Drugs. Heroin hydrochloride was obtained from NIDA and dissolved in $0.9 \%$ sterile saline for i.v. infusions $(60 \mu \mathrm{g} / \mathrm{kg} / 0.1 \mathrm{ml}) . \mathrm{Nal}-$ oxone hydrochloride was obtained from Hospira and dissolved in $0.9 \%$ sterile saline for a s.c. injection in a volume of $1 \mathrm{ml} / \mathrm{kg}$ and $120 \mu \mathrm{g} / \mathrm{kg}$.

Statistics. Heroin self-administration data were analyzed using 2-way ANOVA, with heroin access (ShA versus LgA) as a betweensubjects factor and session or cue (saline versus naloxone paired) as the within-subjects factor. Statistical significance was set at $\alpha=0.05$. Post hoc comparisons were conducted when appropriate, and $P$ values were corrected for multiple comparisons using Bonferroni's method. The statistical analyses for behavioral experiments were performed using GraphPad Prism 7 software. One LgA rat was excluded from the MRI study for failed catheter patency. 
A

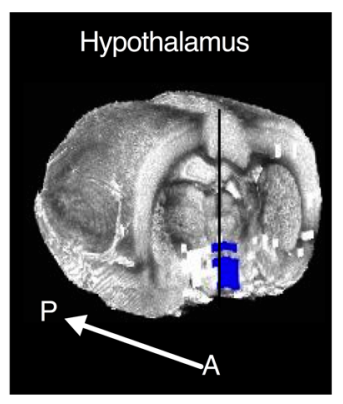

D

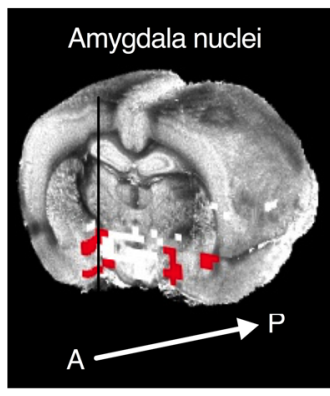

B

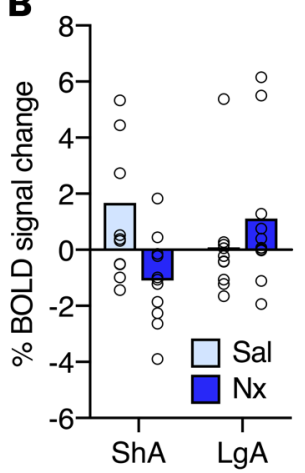

E

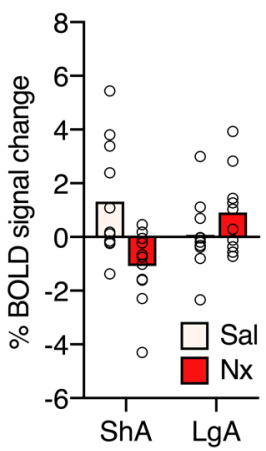

C

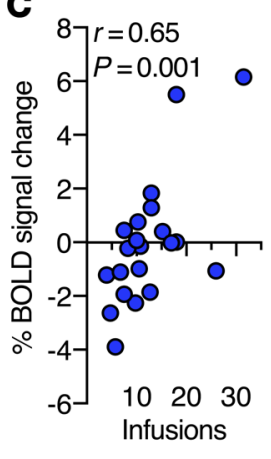

$\mathbf{F}$

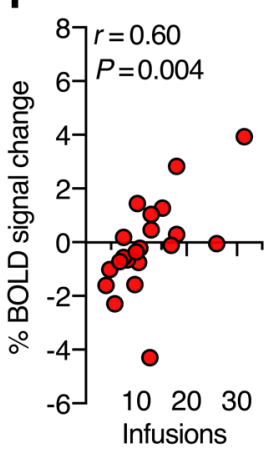

Figure 2. Withdrawal severity during conditioning is associated with changes in the hypothalamic and amygdala nuclei BOLD signal in response to the naloxone-paired cue.

(A) Hypothalamic cluster extracted from the BOLD signal cue $\times$ heroin-access interaction shown in Figure 1C, with a $3 \mathrm{D}$ rendered whole-brain underlay. $\mathrm{A}$, anterior; $\mathrm{P}$, posterior. (B) Mean percentage of BOLD signal change from baseline in the hypothalamic cluster to the cue-only presentations. Dot plot displays individual data for each condition. Sal, saline. (C) Scatter plot of heroin intake during naloxone conditioning and BOLD signal response in the hypothalamic cluster to the naloxone-paired cue across both heroin-access groups (Pearson's correlation). (D) Amygdala nuclei extracted from the BOLD signal cue $\times$ heroin-access interaction shown in Figure 1 , with a $3 \mathrm{D}$ rendered whole-brain underlay. (E) Mean percentage of BOLD signal change from baseline in amygdala nuclei to the cue-only presentations. Dot plot displays individual data for each condition. (F) Scatter plot of heroin intake during naloxone conditioning and BOLD signal response in the amygdala nuclei to the naloxone-paired cue across both heroin-access groups (Pearson's correlation). $n=11$ ShA rats; $n=10 \mathrm{LgA}$ rats.
For fMRI data analysis, the averaged signal from white matter and the ventricles was removed by multiple regression analysis (34, 35 ). We conducted a whole brain 2 (heroin access, ShA versus LgA) $\times 2$ (cue, saline- versus naloxone-paired odor) $\times 2$ (cue presentation block, blocks 1 and 2 versus blocks 3 and 4) mixed-design ANCOVA of the percentage of BOLD signal change, using average respiration rate during the block as a covariate. No a priori regional hypotheses were tested. The 3dClustSim program in AFNI was used to estimate the probability of false-positive clusters, which was used to estimate the cluster size threshold for a given voxel-wise $P$ value threshold to correct for multiple comparisons. A cluster size of 13 with a corrected $P<0.01$ (uncorrected $P<0.01$ ) was considered significant. The correlation between behavior (withdrawal severity as the number of heroin infusions during naloxone conditioning) and BOLD signal (in response to odor cues in each of the 19 ROIs that were extracted from the activation cluster, defined by a significant cue $\times$ heroin-accessinteraction) was evaluated using Pearson's test. $P$ values were corrected for multiple comparisons using FDR $(q=0.05)$.

Study approval. All procedures were reviewed and approved by the NIDA Intramural Research Program's Institutional Animal Care and Use Committee in accordance with the National Research Council Guide for the Care and Use of Laboratory Animals (National Academies Press, 2011).

\section{Author contributions}

SAC, JCMV, and HL conducted the experiments. SAC, RJK, and EGLG analyzed the data. EAS and HL supervised the fMRI experiments. LFV and GFK supervised the behavioral experiments. SAC, GFK, and LFV conceptualized and designed the study and wrote the manuscript. All authors contributed to the manuscript writing.

\section{Acknowledgments}

We thank J. Comins for comments, M. Arends for proofreading the manuscript, T. Ross and H. Gu for technical advice, Y. Shaham for reinstatement experimental advice, B. Priddy and L. Thomas for technical support, and M. Raley (NIDA IRP Visual Media) for graphical abstract support. This work was supported by the Intramural Research Program of the NIDA, NIH, and a postdoctoral fellowship award from the Canadian Institutes of Health Research (CIHR) (FRN 152478 to RJK).

Address correspondence to: Leandro F. Vendruscolo, BRC RM 08A727, 251 Bayview Boulevard, Baltimore, Maryland 21224, USA. Phone: 443.740.2869; Email: leandro.vendruscolo@nih.gov.

SAC's present address is: Center for Adaptive Systems of Brain-Body Interactions, George Mason University, Fairfax, Virginia, USA.
1. Scholl L, Seth P, Kariisa M, Wilson N, Baldwin G. Drug and opioid-involved overdose deaths United States, 2013-2017. MMWR Morb Mortal Wkly Rep. 2018;67(5152):1419-1427.

2. Koob GF, Volkow ND. Neurobiology of addiction: a neurocircuitry analysis. Lancet Psychiatry. 2016;3(8):760-773.
3. O’Brien CP, Testa T, O'Brien TJ, Brady JP, Wells B. Conditioned narcotic withdrawal in humans. Science. 1977;195(4282):1000-1002.

4. Kenny PJ, Chen SA, Kitamura O, Markou A, Koob GF. Conditioned withdrawal drives heroin consumption and decreases reward sensitivity. J Neurosci. 2006;26(22):5894-5900.
5. Carmack SA, Koob GF, Anagnostaras SG. Learning and Memory in Addiction. In: Byrne JH, ed. Learning and Memory: A Comprehensive Reference. Cambridge, MA; Academic Press: 2017: 523-538.

6. Wikler A. Dynamics of drug dependence. Implications of a conditioning theory for research and 
treatment. Arch Gen Psychiatry. 1973;28(5):611-616.

7. Evans CJ, Cahill CM. Neurobiology of opioid dependence in creating addiction vulnerability. F100ORes. 2016;5:F1000 Faculty Rev-1748.

8. Darcq E, Kieffer BL. Opioid receptors: drivers to addiction? Nat Rev Neurosci. 2018;19(8):499-514.

9. Vendruscolo LF, Schlosburg JE, Misra KK, Chen SA, Greenwell TN, Koob GF. Escalation patterns of varying periods of heroin access. Pharmacol Biochem Behav. 2011;98(4):570-574.

10. Schulteis G, Markou A, Gold LH, Stinus L, Koob GF. Relative sensitivity to naloxone of multiple indices of opiate withdrawal: a quantitative dose-response analysis. J Pharmacol Exp Ther. 1994;271(3):1391-1398.

11. Frenois F, Le Moine C, Cador M. The motivational component of withdrawal in opiate addiction: role of associative learning and aversive memory in opiate addiction from a behavioral, anatomical and functional perspective. Rev Neurosci. 2005;16(3):255-276.

12. Sumiyoshi A, Keeley RJ, Lu H. Physiological considerations of functional MRI in animal models [published online ahead of print August 17, 2018]. Biol Psychiatry. https://doi. org/10.1016/j.bpsc.2018.08.002.

13. Liu HS, et al. Dorsolateral caudate nucleus differentiates cocaine from natural reward-associated contextual cues. Proc Natl Acad Sci U S A. 2013;110(10):4093-4098.

14. Paxinos G, Watson C. The Rat Brain in Stereotaxic Coordinates. Cambridge, MA: Academic Press; 2014.

15. Gracy KN, Dankiewicz LA, Koob GF. Opiate withdrawal-induced fos immunoreactivity in the rat extended amygdala parallels the development of conditioned place aversion. Neuropsychopharmacology. 2001;24(2):152-160.

16. Upadhyay J, et al. Alterations in brain structure and functional connectivity in prescription opioid-dependent patients. Brain. 2010; 133(Pt 7):2098-2114.

17. Culpepper-Morgan JA, Kreek MJ. Hypothalamic-pituitary-adrenal axis hypersensitivity to naloxone in opioid dependence: a case of naloxone-induced withdrawal. Metab Clin Exp. 1997;46(2):130-134.

18. Zhou Y, Leri F, Ho A, Kreek MJ. Suppression of hypothalamic-pituitary-adrenal axis by acute heroin challenge in rats during acute and chronic withdrawal from chronic heroin administration. Neurochem Res. 2013;38(9):1850-1860.

19. Koob G, Kreek MJ. Stress, dysregulation of drug reward pathways, and the transition to drug dependence. Am J Psychiatry. 2007;164(8):1149-1159.

20. Koob GF, Schulkin J. Addiction and stress: an allostatic view [published online ahead of print: September 2018]. Neurosci Biobehav Rev. https:// doi.org/10.1016/j.neubiorev.2018.09.008.

21. McCue MG, LeDoux JE, Cain CK. Medial amygdala lesions selectively block aversive pavlovian-instrumental transfer in rats. Front Behav Neurosci. 2014;8:329.

22. Jasinska AJ, Stein EA, Kaiser J, Naumer MJ, Yalachkov Y. Factors modulating neural reactivity to drug cues in addiction: a survey of human neuroimaging studies. Neurosci Biobehav Rev. 2014;38:1-16.

23. Moningka H, Lichenstein S, Worhunsky PD, DeVito EE, Scheinost D, Yip SW. Can neuroimaging help combat the opioid epidemic? A systematic review of clinical and pharmacological challenge fMRI studies with recommendations for future research. Neuropsychopharmacology. 2019;44(2):259-273.

24. Giardino WJ, Eban-Rothschild A, Christoffel DJ, Li SB, Malenka RC, de Lecea L. Parallel circuits from the bed nuclei of stria terminalis to the lateral hypothalamus drive opposing emotional states. Nat Neurosci. 2018;21(8):1084-1095.

25. Huang AS, Mitchell JA, Haber SN, Alia-Klein N,
Goldstein RZ. The thalamus in drug addiction: from rodents to humans. Philos Trans $R$ Soc Lond, B, Biol Sci. 2018;373(1742):pii: 20170028.

26. Canteras NS, Goto M. Connections of the precommissural nucleus. JComp Neurol. 1999;408(1):23-45.

27. Mathis V, Kenny PJ. From controlled to compulsive drug-taking: The role of the habenula in addiction [published online ahead of print: June 2018]. Neurosci Biobehav Rev. https://doi. org/10.1016/j.neubiorev.2018.06.018.

28. Zilverstand A, Huang AS, Alia-Klein N, Goldstein RZ. Neuroimaging impaired response inhibition and salience attribution in human drug addiction: a systematic review. Neuron. 2018;98(5):886-903.

29. Koob GF, Le Moal M. Plasticity of reward neurocircuitry and the 'dark side' of drug addiction. Nat Neurosci. 2005;8(11):1442-1444.

30. Siegel S, Ramos BM. Applying laboratory research: drug anticipation and the treatment of drug addiction. Exp Clin Psychopharmacol. 2002;10(3):162-183.

31. Strang J, et al. Loss of tolerance and overdose mortality after inpatient opiate detoxification: follow up study. BMJ. 2003;326(7396):959-960.

32. Brynildsen JK, Hsu LM, Ross TJ, Stein EA, Yang Y, $\mathrm{Lu} \mathrm{H}$. Physiological characterization of a robust survival rodent fMRI method. Magn Reson Imaging. 2017;35:54-60.

33. Lu H, Zou Q, Gu H, Raichle ME, Stein EA, Yang Y. Rat brains also have a default mode network. Proc Natl Acad Sci U S A. 2012;109(10):3979-3984.

34. Cox RW. AFNI: software for analysis and visualization of functional magnetic resonance neuroimages. Comput Biomed Res. 1996;29(3):162-173.

35. $\mathrm{Lu} \mathrm{H}$, et al. Registering and analyzing rat fMRI data in the stereotaxic framework by exploiting intrinsic anatomical features. Magn Reson Imaging. 2010;28(1):146-152. 